\title{
Jóvenes y cultura audiovisual: nuevos modos de ver televisión
}

\author{
Young people and audiovisual culture: new ways of watching television
}

\section{Jovens e cultura audiovisual: novos modos de ver televisão}

\author{
Rosario Sánchez Vilela \\ Doctora en Ciencia Política (UDELAR), Magister en Comunicación Social (UCU), Profesora Titular e Investigadora de la \\ Universidad Católica del Uruguay. Integra el Sistema Nacional de Investigadores de ANII, Uruguay. \\ <rsanchez@ucu.edu.uy; rsanchezvilela@gmail.com>
}

\section{RESUMEN}

Esta comunicación se propone revisar algunos de los conceptos teóricos que hasta hace poco han sido clave para comprender los procesos de recepción, particularmente los vinculados a la televisión. Se trata de una discusión que forma parte de las etapas de un proyecto de investigación orientado a estudiar la cultura audiovisual de los jóvenes uruguayos en tiempos de convergencia mediática y el lugar de la televisión en ella. ¿Qué es ver televisión para los jóvenes? ¿qué significados tiene? ¿en qué narrativa de la cotidianidad se integra? Estas son algunas de las preguntas que articulan el proyecto. Su respuesta supone reconsiderar las nociones de mediaciones, domesticidad y domesticación. El hogar y lo doméstico ¿han dejado de tener relevancia en los nuevos modos de ver televisión? Se avanzarán aquí algunos resultados a partir de entrevistas semiestructuradas focalizadas en las prácticas de los jóvenes en la construcción de su vida cotidiana.

\section{ABSTRACT}

This article attempts to examine some of the theoretical concepts that, until recently, have been crucial for the understanding of TV-related reception processes. The present discussion belongs to a reseach project which focuses on audiovisual culture in Uruguayan young people during times of media convergence, and the place of television within such process. What is to watch TV, for the young? What does it mean? In which narrative of everyday life is this activity integrated? These are some of the questions that articulate the project. Answering them will envolve a reconsidaration of the notions of mediation, domesticity, and domestication. Have home-related and the domestic aspects lost their relevance in the new ways of watching television? Some early results have been rendered by a series of semi-structured interviews which focus on how young people construct their everday practices.

Keywords: Television reception. Communication. Convergence

\section{RESUMO}

Este artigo se propõe a revisar alguns dos conceitos teóricos que até recentemente têm sido chave para a compreensão dos processos de recepção, particularmente, os relacionados com a televisão. Esta é uma discussão que integra as etapas de um projeto de pesquisa que visa estudar a cultura audiovisual de jovens uruguaios em tempos de convergência midiática e o papel ocupado pela televisão. O que significa ver televisão para os jovens? Quais os seus significados? Em qual narrativa cotidiana está integrada? Estas são algumas das perguntas que articulam o projeto. Sua resposta envolve repensar as noções de mediações, domesticidade e domesticação. As relações domésticas com o lugar? Perderam a sua relevância a partir das novas formas de ver televisão? Alguns resultados foram apresentados a partir de entrevistas semiestruturadas dirigidas as formas com que os jovens constroem a sua vida cotidiana. 


\section{Introducción}

Esta comunicación se propone la revisión de algunos de los conceptos teóricos que desde mediados de los años ochenta han sido puestos en juego para comprender los procesos de recepción, particularmente los vinculados a la televisión. Es esta una discusión que forma parte de las etapas de un proyecto de investigación que se propone estudiar la cultura audiovisual de los jóvenes uruguayos en tiempos de convergencia mediática y el lugar de la televisión en ella. La investigación se concentrará en jóvenes entre 18 y 24 años de la capital del país, Montevideo. El proyecto se asume como un primer abordaje que podrá extenderse a otros jóvenes y zonas del país en investigaciones futuras. Es esta una primera aproximación a una temática amplia y compleja, la cultura audiovisual de los jóvenes, en un aspecto específico y acotado: sus modos de ver televisión.

¿Por qué ocuparse de los jóvenes? Según los datos proporcionados por Ibope Media-Uruguay los jóvenes entre 18 y 24 años constituyeron en 2013 el 11,45\% del total del universo y entre 25 a 34 años el 16,1\% y en 2014 alcanzan el $11,37 \%$ y $15,87 \%$ respectivamente ${ }^{1}$. Paralelamente el crecimiento de la penetración de Internet es constante². Se podría pensar que los jóvenes se han retirado del consumo televisivo, sin embargo miran a Peter Capusotto en Youtube o un capítulo que alguien "colgó" en el Facebook, se bajan temporadas enteras de series y las ven en la tablet, la computadora o el celular. En suma, siguen viendo televisión, pero no en el artefacto integrado al mobiliario familiar desde mediados del siglo XX. Miran televisión de otro/s modo/s. Ver televisión tiene otro significado para ellos.

¿Qué es ver televisión para los jóvenes? ¿qué significados tiene? ¿en qué narrativa de la cotidianidad se integra? ¿Cómo se ha transformado su relación con el hogar, el espacio doméstico y las temporalidades de la vida diaria (el tiempo

1 No son muchos los datos de consumo de medios respecto al rango de edad específico del que se ocupa la investigación. No obstante, la información proporcionada por la encuesta de Radar sobre el Perfil del Estudiante Universitario 2014, constituye un marco de referencia para nuestro trabajo. Según esta encuesta el $24 \%$ de los estudiantes universitarios no mira televisión, el 26\% lo hace hasta 1 hora diaria, el $28 \%$ de 1 a 2 horas y el $13 \%$ de 2 a 3 horas. El $32 \%$ declara que sigue algún programa y de ellos el $80 \%$ sigue series televisivas. Cabe señalar que la encuesta abarca tramos de edades más amplios en tanto incluyó a estudiantes de posgrado. Disponible en: < http://www.prouniversitarios.com/Encuesta Perfil del Estudiante Universitario 2014.pdf>. Acceso en: 4 de oct. 2014.

2 La información que provee la encuesta de Imaginarios y Consumo Cultural (2009 y 2014) proporciona datos generales de consumo en Uruguay que indican que en 2009 el 34\% de la población usa Internet todos los días y en 2014 aumente a 47\%. Según el Perfil del Internauta 2014 (Radar, 2014) la penetración de Internet en el segmento de 12 a 19 años alcanza el 96\%, lo que se señala como efecto multiplicador Plan Ceibal. Sin embargo, no son muy abundantes los datos específicos en el tramo de edad que aquí nos proponemos estudiar. Disponible en: < http://www.gruporadar.com.uy/01/wp-content/ uploads/2014/12/El-Perfil-del-Internauta-Uruguayo-Resumen-ejecutivo.pdf >. Acceso en: 19 .dic. 2014. 
del ocio, el del trabajo, el del estudio)? Estas son algunas de las preguntas que articulan el proyecto y que obligan a reconsiderar a la televisión como tecnología, su articulación con la vida cotidiana, la dimensión de la domesticidad y de la domesticación como claves de comprensión de los sentidos de ver televisión.

El estudio de estos procesos ha estado centrado fundamentalmente en la familia y el hogar como contexto de recepción (Morley, 1996, 1992; Silverstone, 1996, 2004; Silverstone e Hirsch, 1996; Lull, 1990). En una comprensión centrada en las prácticas de los jóvenes en la construcción de su vida cotidiana, el hogar y lo doméstico ¿han dejado de tener relevancia en los nuevos modos de ver televisión? Todo ello hoy debe ser repensado a la luz de las nuevas tecnologías. Intentaré aquí recuperar y reformular los aspectos vigentes de aquellas ideas que fueran reveladoras de una perspectiva comprensiva de los fenómenos comunicativos y culturales en torno a la televisión. Se avanzarán en este texto algunas reflexiones a partir de una primera aproximación, mediante entrevistas semiestructuradas, a la relación entre jóvenes y consumo de ficción televisiva.

\section{El punto de partida: la tradición de los estudios culturales}

La tradición de los estudios culturales constituye la caja de herramientas, para pensar los nuevos modos de ver televisión. Es que la preocupación por el papel de la televisión en la vida cotidiana encuentra allí sus antecedentes más relevantes, especialmente en las investigaciones de David Morley $(1996,2008)$ y Roger Silverstone $(1996,2004)$ que estudiaron las prácticas de ver televisión en el ámbito doméstico. Esta perspectiva sostiene que las tecnologías no permanecen inmutables en su encuentro con lo cotidiano y que su sentido se define en relación al conjunto de discursos de la vida cotidiana. Es desde este punto de partida que la relación con el tiempo y las rutinas del diario vivir, las relaciones de género y generacionales en el hogar son consideradas fundamentales para comprender los procesos de apropiación y de domesticación de la televisión tradicional.

Uno de los aportes más significativos del giro hacia las audiencias en los estudios culturales fue precisamente el reconocimiento de que los contextos de recepción son relevantes para comprender los significados que se producen tanto sobre un programa o contenido como sobre la propia tecnología. Lo doméstico fue entonces identificado como el ámbito "natural" de ver televisión $y$, como se ha dicho, el énfasis estaba puesto en la familia.

Es decir, la domesticidad no solo se asociaba al hogar sino a la existencia de la familia. La audiencia de la televisión ha sido comprendida así como una audiencia situada en un determinado espacio en el hogar: el living, la sala de estar, lugar del que desplazó a la radio. Incluso con la proliferación de aparatos 
de televisión en el hogar, la audiencia continuó siendo una audiencia situada, con una relación estable y acotada tanto con el espacio como con el tiempo.

Desde esta perspectiva el ver televisión se integraba en un entramado de prácticas estructuradas que configuraban el tejido de la vida cotidiana. Las emisiones de la televisión se inscribían en las rutinas diarias en una relación dialógica con los ritmos domésticos. La programación de la televisión tradicional sabía que debía adaptarse a ellos.

Propongo aquí recuperar esta mirada que autores como Silverstone y Morley propusieron en las décadas del 80 y 90, para ponerla en juego al estudiar hoy los nuevos modos de ver televisión. Considero que sigue vigente la idea de que los sentidos de ver televisión necesitan ser investigados en el conjunto de las prácticas cotidianas y domésticas, aunque ahora quizás la familia haya perdido centralidad. Pero la vida cotidiana continúa siendo una mediación neurálgica, porque como señalan Berger y Luckmann, ella es realidad imperiosa:

\footnotetext{
Entre las múltiples realidades existe una que se presenta como (...) "suprema realidad". La tensión de la conciencia llega a su apogeo en la vida cotidiana, es decir, ésta se impone sobre la conciencia de manera masiva, urgente e intensa en el más alto grado. Es imposible ignorarla y aún más difícil atenuar su presencia imperiosa. (...) Experimento la vida cotidiana en estado de plena vigilia. Este estado de plena vigilia con respecto a existir y aprehender la realidad de la vida cotidiana es para mi algo normal y evidente por sí mismo, vale decir, constituye mi actitud natural (Berger y Luckmann, 1979, p. 39).
}

Estecarácterimperiosodelavidacotidianaesválidoyparticularmentepotente paralosjóvenesquevivenlatensiónentreamoldarseaunacotidianidadyaconstruida y la necesidad de diferenciarse, marcar su identidad, romper con lo establecido.

La premisa de fondo de este abordaje consiste en considerar que la significación y el poder de un medio solo pueden ser comprendidos en su relación con la multiplicidad de discursos de la cotidianidad. Ellos no se explican en forma absoluta desde el medio y sus rasgos tecnológicos, tampoco desde sus contenidos. Las significaciones que una tecnología de la comunicación, un medio específico, o un producto televisivo adquiere en la vida de las personas son fruto de un entramado de interrelaciones, más o menos sobredeterminantes. En ese sentido Silverstone afirma:

Tenemos que considerar a la televisión como un medio inserto en los múltiples discursos de la vida cotidiana. $Y$ tenemos que entender qué son esos discursos, cómo se determinan, cómo se entrelazan y, lo que es más importante, cómo se los debe distinguir desde el punto de vista 
de su mutua influencia relativa. Esta tarea de descripción y análisis exige tanto la atención teórica como la empírica (Silverstone,1996, p. 12).

En esta misma dirección, uno de los aportes de los estudios culturales ha sido su advertencia respecto a la ausencia de homogeneidad en el significado de ver televisión y el señalamiento de que, de manera más precisa, deberíamos hablar de modalidades diversas de ver televisión.

\section{Una primera aproximación a los nuevos modos de ver televisión}

La televisión ya no es lo que era y sus receptores tampoco. Entre otros cambios, uno de los más relevantes es que el hogar como contexto tradicional de recepción ha diluido su dominancia: hoy la televisión se puede ver en la tablet, en el celular, en la laptop, esto es, en contextos diversos y cambiantes. Aunque el hogar continúa siendo un lugar de recepción de la televisión, hace tiempo ya que la multiplicidad de pantallas -primero por la multiplicación de los aparatos en la casa, luego por la incorporación de nuevas tecnologías- lo ha reconfigurado como contexto de recepción. Se ha producido un desarrollo de la autonomía del consumo a la vez que se rearticulan las dinámicas familiares y domésticas. Este es el núcleo central de nuestro asunto.

Brevemente: por autonomía del consumo me refiero a las prácticas de ver televisión que se independizan del momento de emisión, pero también a la autonomía respecto a los otros espectadores. No solo se puede mirar la televisión sin estar en simultaneidad con los otros y es más fácil apartarse de mirar lo que mira la familia, sino que también fuera de la familia se exacerba la posibilidad de diferenciarse del vecino o de las personas con las que uno se encuentra, por ejemplo, en una misma sala de espera del médico. Esta breve caracterización ya incluye algunos de los rasgos de los nuevos modos de ver televisión que se manifiestan en los jóvenes, aunque no exclusivamente en ellos.

Uno de los aspectos más relevantes es el cambio en la relación con el tiempo y con el espacio. En los jóvenes, más que en otros espectadores, el ver televisión se independiza del artefacto y ello define la transformación de la relación con el espacio. Se produce una cierta deslocalización de la práctica que permite un consumo deambulante de la televisión, por ejemplo, mientras se viaja hacia el centro de estudios o hacia el trabajo, cuando se está en una sala de espera, etc. Sin embargo, el hogar, la casa continúa siendo el lugar que se menciona en primera instancia al referirse al consumo de productos televisivos (aunque también allí se puede deambular por sus diferentes espacios), mientras 
que, escuchar música se reconoce como lo más habitual cuando se está en tránsito por la ciudad.

El cambio en relación con el tiempo tiene como consecuencia una transformación de la expectativa y de la experiencia de la serialidad. La televisión en lógica de broadcasting implica la organización temporal en sucesión de los contenidos hacia un público amplio que estará ante la pantalla en simultáneo. La espera del próximo capítulo ha formado parte de la fruición del consumo tanto de la radio como de la televisión. Así mismo, la teledramaturgia en sus diferentes formas ha explotado esta dimensión trabajando con el suspenso, el "gancho" al final del capítulo, etc. En cambio, en los nuevos modos de ver televisión ya no se espera el próximo capítulo de la misma manera, organizando el tiempo en relación al momento definido en la programación por el emisor. No obstante, la expectativa no ha desaparecido sino que ha cambiado sus formas. Se podría ensayar una tipología de esperas. Para algunos, es la espera del momento fijado en la rutina propia para ver, por ejemplo, una serie: se trata un momento definido desde el consumidor dentro de sus rituales cotidianos y que puede ser cambiado. Para otros, persiste la expectativa ansiosa en relación a la disponibilidad de la próxima temporada, o están pendientes del momento en que saben que se emitirá el siguiente capítulo y es entonces que se disponen a verlo, reeditando el ver televisión clásico de la televisión abierta: "Estabamos esperando que cada lunes colgaran el capítulo y ahí nos poníamos a verlo" (Mauricio, estudiante 24 años).

También pautando una nueva relación con el tiempo, uno de los modos de ver televisión que se observa es aquel que podríamos llamar, metafóricamente, bulímico. Con modo bulímico, me refiero a un tipo de consumo compulsivo, en atracones de capítulos de una serie determinada, en sesiones extensas de visionado. En este modo de ver el producto televisivo, se manifiesta tanto la dificultad para detener el visionado, como la sensación de vacío del fin. El siguiente testimonio puede ilustrar esta idea: "Se me hacían las cuatro de la mañana viendo capítulos y cuando terminé de ver la última temporada... ¡Y ahora qué voy a hacer!" (Sofía, estudiante, 23 años). Esta práctica suele darse en soledad o en pareja, pero en general no involucra a la familia o al grupo de amigos. ${ }^{3}$

La autonomía en el consumo redefine la mediación de la familia y también la de los pares. No quiere decir esto que ella desaparezca del proceso de generación de sentido de un texto televisivo específico e incluso de la elección

3 Es pertinente señalar que este tipo de consumo no es exclusivo del visionado de series, sino que guarda semejanzas con comportamientos de consumidores de diversa literatura: lectores que no pueden detener la lectura de una novela; la lectura ávida de revistas, comics, etc. 
del producto que se elige ver, pero lo que se pierde es la co-presencia de discursos interpretativos en el momento mismo de contacto con el programa. No obstante, la conversación mantiene su lugar en la recepción: las series se recomiendan entre amigos; los hijos las sugieren a los padres, se comentan y se discuten. La reelaboración de los relatos en la conversación continúa siendo parte de los procesos de apropiación (Thompson, 1995), pero la reducción de la instancia colectiva, reconfigura la sociabilidad dentro y fuera del hogar (Livingstone, 2007, p. 319).

Al ser interrogados sobre si ven televisión, los jóvenes suelen decir que no ven televisión, que no les gusta o, en algunos casos en los que se han ido a vivir solos declaran, casi de manera desafiante, que no tienen televisión:

No, no veo....Veo televisión cuando de repente voy a lo de mis padres que siempre está la tele prendida y ahí vemos algo y comentamos. (Florencia, estudiante, 22 años)

No, yo no veo televisión, bueno ta...si estamos cenando en familia ahí sí...(Pablo, estudiante, 20 años)

Estas expresiones son ilustrativas de que para ellos el ver televisión está ligado al artefacto, mientras que cuando los contenidos televisivos se ven por Internet adquieren otro estatus. No obstante, también se manifiesta que, junto a los nuevos modos de ver televisión, persisten algunas viejas prácticas que parecen adquirir un significado de concesión a la familia; una familia fragmentada en la que convergen diversos estilos de vida de los que los modos de ver televisión son solo una expresión.

Las transformaciones hasta aquí señaladas están ligadas directamente a un cambio en la televisión como tecnología: la televisión ya no está adherida a un artefacto, sino que circula por diferentes aparatos con lo que se altera su lugar y significación en la domesticidad. La televisión, que fue clave para la venta de otras tecnologías, se funde ahora con ellas y expande su capacidad de articulación con el sistema tecnológico. Me refiero a que aquellos artefactos que se venden por televisión (celulares, tablets, laptops) son ahora también su vehículo. En otras palabras: la televisión sigue siendo hoy un artefacto, pero lo trasciende y se define más por las características de sus contenidos que por la materialidad del objeto. Una serie como Los Soprano o Friends sigue siendo un producto televisivo, se vea en la tablet o en la pantalla de un televisor. La operación de consumo está ahora más ligada al producto. La relación con el texto ocupa un lugar central, a diferencia de lo que ocurría en el marco del flujo 
televisivo. La elección del programa es equivalente a una acción de compra en un menú de ofertas en el que el espectador elige qué ver. En cierta manera, el recorrido por la oferta de Netflix, por ejemplo, reedita la vieja práctica del desplazamiento de la mirada por las góndolas del video club hasta elegir una película.

\section{A modo de cierre}

"Lo doméstico no es una categoría exenta de problemas: los hogares no son las familias, las familias van más allá del hogar", advertía Morley (1996, p. 294). La noción de domesticidad está circunscripta al origen latino del término, domus, casa. La dimensión doméstica de la cotidianidad es la que se desenvuelve en el ámbito del hogar, el espacio privado donde se vive y puede incluir o no a la familia. Es posible una domesticidad sin familia, de quien vive solo, o en combinaciones de convivencia (jóvenes que se juntan para compartir gastos, independizándose de sus familias, o que emigran y comparten la casa con personas que apenas conocen y que pertenecen a culturas diversas). Otra es la domesticidad que se teje en el hogar con familia. Aquí los lazos de parentesco, las relaciones afectivas y la historia familiar construyen otra cara de la cotidianeidad, más allá de la conexión específica con "la casa", trascendiendo sus límites (Maronna y Sánchez Vilela, 2006, p. 96). En su sentido sustancial habría que entender lo doméstico como aquel entramado de prácticas que conforman la idea de mundo propio, controlado por mí, privado, seguro y previsible, en suma, hogar, con o sin familia.

Ligado a la consideración de lo doméstico se encuentra el concepto de domesticación para explicar nuestra relación con las tecnologías. Por domesticación de la tecnología se entiende

\footnotetext{
la capacidad de un grupo social (una casa, una familia, pero también una organización) para apropiarse de los artefactos tecnológicos y los sistemas de transmisión e incorporarlos a su propia cultura - sus propios espacios, sus propios tiempos, su propia estética y su propio funcionamiento-, para controlarlos y para hacerlos más o menos "invisibles" dentro de las rutinas diarias (Silverstone, 1996, p.169).
}

El proceso supone la decisión de compra, el ingreso al hogar y la definición de una ubicación en la vida diaria.

Propongo aquí la idea de que la domesticación en los nuevos modos de ver televisión de los jóvenes implica decisiones en una doble articulación: la elección de dispositivos que permiten la movilidad y la elección de programas/ 
contenidos televisivos específicos que se insertan en la tarea de construcción de su vida cotidiana. En este sentido, la domesticación es el proceso por el cual el ver televisión se constituye en una marca de identidad y supone un proceso de construcción de estilo de vida diferenciado.

Uno de los aspectos que conforma el placer de ver televisión en los jóvenes es la posibilidad de ubicar el consumo en el tiempo que ellos deciden, con una organización que rompe las rutinas tradicionalmente marcadas por la familia: un tiempo para comer, un tiempo para ver televisión, un tiempo para dormir y en el que la casa queda en silencio. En los nuevos modos de ver televisión la simultaneidad se rompe, ya no es condición necesaria para poder ver un programa y ello se experimenta como libertad de construcción de un estilo de vida propio, de organización de una rutina en paralelo a la del resto de la familia. Podríamos hablar de una domesticidad paralela, que se construye en la articulación de varias tecnologías para diferentes usos y en diferentes consumos. Me refiero a que por diferentes artefactos (celular, computadora, tablet, etc) el juego en red, el mensajeo constante en WhatsApp, el chat por redes sociales, ver películas o series televisivas, los jóvenes configuran un mundo privado de prácticas cotidianas, una suerte de burbuja propia dentro del hogar. Los nuevos modos de ver televisión se insertan en este conjunto de prácticas diferenciadas, como un recurso más en la construcción de un mundo propio y distintivo en el que la relación con la televisión está centrada en el texto televisivo y no en el artefacto.

\section{REFERENCIAS}

BERGER, Peter; LUCKMANN, Thomas. La construcción social de la realidad. Buenos Aires: Amorrortu, 1979.

Grupo Radar. El Perfil del Estudiante universitario 2014. Disponible en: <http:// www.gruporadar.com.uy>. Acceso en: 4.oct.2014.

Grupo Radar. El Perfil del Internauta uruguayo 2014. Disponible en: <http://www. gruporadar.com.uy>. Acceso en: 19.dic.2014.

LIVINGSTONE Sonia, From family television to bedroom culture: Young people's media at home, en DEVEREUX, Eoin (Ed.). Media Studies: Key issues and Debates. Londres: Sage, 2007.

LULL, James. Inside Family Viewing: Ethnographic Research on Television Audience. Londres: Routledge, 1990. 
MARONNA, Mónica; SÁNCHEZ VILELA, Rosario. La puesta en relato de lo cotidiano, en RICO, Carmen (org.) Relecturas de Michel de Certeau. México: Universidad Iberoamericana, 2006.

MORLEY, David. Televisión, audiencias y estudios culturales. Buenos Aires: Amorrortu, 1996.

Medios, modernidad y tecnología. Hacia una teoría interdisciplinaria de la cultura. Barcelona: Gedisa, 2008.

OROZCO, Guillermo. Televisión, audiencias y educación. Buenos Aires: Norma, 2001.

SÁNCHEZ VILELA, Rosario. Sueños Cotidianos. Telenovela y oralidad. Montevideo: Taurus-UCU, 2000.

SILVERSTONE, Roger; HIRSCH, Eric. Los efectos de la nueva comunicación. EI consumo de la nueva tecnología en el hogar y en la familia. Barcelona: Bosch, 1996.

SILVERSTONE, Roger. Televisión y Vida Cotidiana. Buenos Aires: Amorrortu. 1996.

Por qué estudiar los medios. Buenos Aires: Amorrortu, 2004.

THOMPSON, John B. Los media y la modernidad. Una teoría de los medios de comunicación. Buenos Aires: Paidós, 1995.

Recebido em: 23/11/15

Aceito em: 8/12/2015

Endereço da autora:

Rosario Sánchez Vilela <rsanchez@ucu.edu.uy; rsanchezvilela@gmail.com>

Universidad Católica del Uruguay

Sede Central | Montevideo

Av. 8 de Octubre 2738, entre Garibaldi y Estero Bellaco

Montevideo, CP 11600 